\title{
Overall Feasibility of Low Cost Conversion from PV to PVTw
}

\author{
E. Rebollo, F. R. Blánquez, I. López and C. A. Platero,
}

C. Carrero

\begin{abstract}
Photovoltaic modules are more efficient the lower their temperature is. Photovoltaic-thermal technologies, PVT, exploit this feature by refrigerating the conventional photovoltaic module with a heat exchanger, usually based either on air or on water. The water is heated in the exchanger, so it can be used as hot water directly, saving energy.

However, achieving high electrical and thermal efficiency requires specifically designed panels with the exchangers built in. This increases the cost and generally puts them out of the market.

In this paper we study the possibility of building profitable generic exchangers which could be fit onto standard photovoltaic panels. In order to compensate for their low performance, they are operated allowing the water to heat a few degrees only. It would have to be heated further for domestic use, but ensures that the photovoltaic panel works at a low temperature and high electrical efficiency. A coarse estimation of the simple payback period in several scenarios is made, concluding that the energy savings in preheating water and the extra electrical production would pay for the cost of the exchangers in less than two years.
\end{abstract}

\section{INTRODUCTION}

It is a well known property of photovoltaic, PV, modules that their efficiency in the production of electricity is higher the lower their operating temperature is. For efficiency it is understood the ratio of the energy arriving to the panel in the form of radiation which is actually converted into electricity. There exist many research works describing the temperature-efficiency dependence in specific cases; a survey of mathematical correlations can be found in [1] and [2].

A photovoltaic module is formed by groups of seriallyconnected photovoltaic cells. Sometimes there are several groups connected in parallel. The number of cells per group and the number of groups in a panel depends on the make, the electrical specifications and the size of the panel. The power that a single cell may deliver depends on its working temperature; the cooler, the greater. Therefore, the maximum power that a group of cells may deliver is given by the temperature of its cells. The electrical effects of this over the performance of the panel are analyzed in [3], as well as the performance of different cooling strategies at cell level.

This work shall be analyzing the whole panel, abstracting its inner electrical phenomena. The effect of cooling the panel by fitting a low cost heat exchanger on its back shall be studied. A conventional panel may achieve electrical efficiencies of typically no more than $15 \%$ and lower than $25 \%$ in the best of cases for laboratory prototypes [4]; this still leaves a loss between 75 and $85 \%$. By cooling the panel with a fluid, typically either air, water or both, the electrical efficiency will increase and the heat absorbed by the coolant may also be used. With this double usage, the global energetic efficiency (electrictthermal) might achieve values over $70 \%$ for a well-built system [5]. These systems are generically called photovoltaic thermal systems, PVT, adding the appendix ' $w$ ' for the ones based on water or ' $a$ ' for the ones based on air. An overview including key design aspects, a summary of researchers' experience and recommendations and a brief theoretical summary of this technology can be found in [6].

The way in which the heat exchanger forming the cooling circuit is arranged on the PV panel admits a variety of alternatives. A detailed review of the state of the art [7] distinguishes two main categories of PVT designs: channel above $P V$ and channel below $P V$. In this work it is studied the case of water. Situating the exchanger above the PV panel has some drawbacks. First, it forces to use a transparent exchanger which may be subject to mechanical stress, therefore requiring a careful design and selection of materials [8]. Second, it has been found that it may reduce electrical efficiency by around $10 \%[7]$

On the other hand, the main problem with channel below $P V$ designs is achieving a good heat transfer between the panel and the exchanger. The topology of 'sheet and tube' is the most direct design: a sheet of good thermal conductor is glued to the back of the panel and the tubes for the fluid are put in contact with it. The free side is thermally isolated. In spite of the simplicity of the design, it has two major problems. Achieving a good contact between the sheet and the panel on one side and between the tubes and the sheet on the other is not straightforward [9]. An alternative is the box channel configuration, in which the exchanger is formed by rectangular tubes glued to the back of the panel, also studied in [9]. This is the one chosen for this work.

The problem of achieving a sufficient heat transfer with any of the configurations has lead Zakharchenko [10] to recommending to discard adapting existing PV panels and to adopt specifically designed, integrated PVT panels. In spite of this, this work is entirely focused on adapting existing panels for reasons which shall be commented following.

It is pointed out in [6] that the strategic advantages of PVT technology are:

- its dual heat-electricity purpose,

- its efficiency and flexibility,

- its wide application, 
- that it is cheap and practical and

- it can easily be retrofitted/integrated without major modification.

Among these points, research and development has centered on increasing the global efficiency. Standard-based solutions fall far from the optimum performance, but we do consider they should be studied a little further before ruling them out. In fact, they stress several potentials of the technology: low price, flexibility and contribution for reducing the payback period. For some purposes, these factors may be critical. In fact, the Roadmap published through the PVT Forum [11], cites as a challenge achieving plug-and-play installation of PVT, and the domestic sector as having the largest market potential. The potential of small industries must be added, some equipped with non-negligible photovoltaic infrastructures on roofs.

This text proposes to shift the point of view from optimality towards flexibility and cost of PVT. It is intended to find out if retrofitting a thermal device onto an existing installation may prove interesting. The performance of a low cost adaptation of a standard, also low cost P-Si panel is analyzed. This will allow building a basic idea of the expectable advantages and the potential problems of designing this kind of systems.

The study is planned in a conservative scenario in terms of performance, materials and design. The photovoltaic panel used for the experiments is low cost and low perfornance. Materials used for the adaptation are standard. The engineering solutions adopted are applicable to a generic panel. This should result in a low performance heat exchanger and in many constructive problems. Moreover, at a number of points in the experience, the cheapest alternative was adopted.

Section II summarizes the objectives of the study and the thermal problem. Section III is dedicated to describing the three alternative designs which were actually built and their constructive problems. Section IV describes the experiments carried out with the definitive prototype and its performance is analyzed in section V. Cost is discussed in section VI, including some interesting extrapolations of the studied case. Finally, section VII summarizes our conclusions.

\section{OBJECTIVES AND BASIC IDEA}

In technical terms, the study has two objectives:

- To increase the electrical efficiency of a conventional photovoltaic panel

- To obtain thermal energy in the form of pre-heated water.

The device will always be conceived as a complement to an existing water heating installation, not as a proper heating system by itself.

The thermal problem associated to the heat exchanger must be explained. The heat exchanger that will result from the low-cost criteria mentioned in section I will achieve a poor performance. Therefore, for a viable solution, any design must be based necessarily on a very small temperature increase in the cooling water, both for electrical efficiency and thermal energy:

$$
\begin{aligned}
& Q=U A \Delta T_{L M T D} \\
& Q=\dot{m}_{w} c_{p, w} \Delta T_{w}
\end{aligned}
$$

Equation (1) represents the well-known heat transfer model for a heat exchanger [12], in which $Q$ is the power interchanged through the area of contact, $U$ is the heat transfer coefficient, $A$ is the area of contact and $\triangle T_{L M T D}$ is the log-mean temperature difference, LMTD, which represents an average temperature drop between the surface of the PV module and the cooling water. Equation (2) represents the heat absorbed by the cooling water as it passes through the exchanger, in which $\dot{m}_{w}$ is the water mass flow, $c_{p, w}$ is its specific heat and $\Delta T_{w}$ is its temperature increment from inlet to outlet. Naturally, both expressions must be equal.

In summary, the thermal problem of the low cost solution (low $U$, discussed in section III) is compensated for by a large $\Delta T_{L M T D}$ in order to make $Q$ as large as possible. For this, a low $\Delta T_{w}$ is necessary, which forces to operate with large mass flows, $\dot{m}$, in (2).

As a result, it is expected that the adapted panel will show a significant increase in electrical efficiency due to the low panel temperatures, but a low thermal efficiency due to the small $\Delta T_{w}$.

From the point of view of viability, it is evaluated if the increment of electrical efficiency and the amount of thermal power obtained could justify adding a heat exchanger to existing panels.

\section{MATERIALS, PROTOTYPES AND CONSTRUCTION}

The decision was made to build a channel below $P V$ panel with box channel exchanger. The choice between a sheet and tube topology and the channel box one, widely regarded as economically advantageous [6][7], was made for two reasons:

1) The box maximizes area of contact.

2) The channel itself is in contact with the back of the panel, which eliminates the problem of binding the tubes to the sheet.

The heat exchanger was therefore similar to the one in [9]. Two design possibilites for the heat exchanger were discarded before arriving to a feasible one.

The system parted from a commercial glazed, P-Si, 12 V, 7 W, 36 cell PV module. Its dimensions were 425 by $300 \mathrm{~mm}$.

\section{A. Previous prototypes}

In order to maximize heat transfer between panel and water, it was intended to let the water flow directly in contact with the back of the panel. This would have also been advantageous regarding the weight and simplicity of the system. Weight would not appear to be a major issue in this prototype in any case because of its small size, but it could become a problem with larger panels.

The first prototype (figure 1, left and middle) consisted in a $11 \mathrm{~mm}$ thick slab of polyethylene which had four milled water conductions, $5 \mathrm{~mm}$ deep and $45 \mathrm{~mm}$ wide. At the ends 

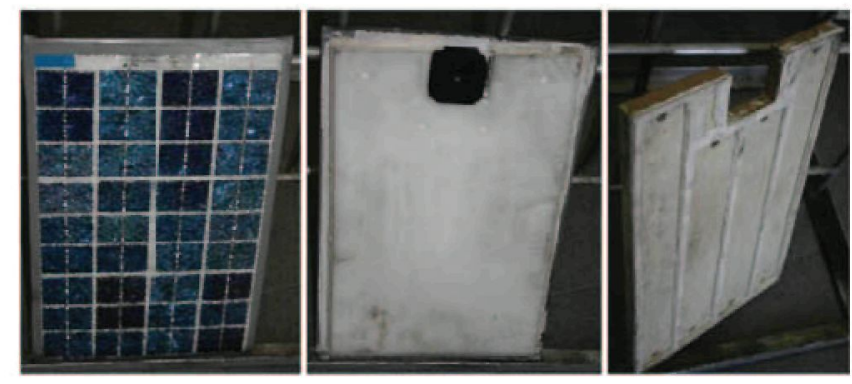

Figure 1. Broken glass of the polyethylene slab prototype and heat exchanger at its back (left and middle). Epoxi resin heat exchanger (right).

of each there were fast valves, the bottom ones as inlets and the top ones as outlets (see section IV). It was assumed that the material would provide sufficient insulation, so nothing was added to the free side of the slab.

The second prototype (figure 1, right) had a similar geometry but the material was changed to epoxy resin, moulded to fit the back of the panel perfectly. It was also attached to its back with silicone. The corresponding holes were left for fixing the fast valves as in the previous design. It was also considered that the insulation that the resin would provide would be sufficient.

Two main problems appeared during the building of the prototypes. First, the mechanical forces due to the water pressure in the exchanger (connected to the mains), the weight of the water and thermal expansions and contractions were not negligible. The union of the panel with the exchangers of the first two prototypes soon developed leaks. Fitting a device to press the exchanger against the panel improved the situation but finally ended by breaking the module.

The idea of having direct contact between the cooling water and the panel was abandoned because a valid design would necessarily fall out of a low-cost approach.

Second, it was found that achieving a reasonable thermal contact between the surface of the exchanger and the back of the panel was not straightforward, in line with the remarks pointed out by Zakharchenko [10] and Chow [13]. The material used for the exchanger needs to be a good thermal conductor, but the lack of flatness and evenness of the back of the PV module would make it difficult to attach any exchanger properly. The first prototype showed that commercial polyethylene slabs were not flat enough either, which penalized both mechanical resistance and thermal contact.

\section{B. Final prototype}

The final exchanger was formed by four parallel pieces of standard aluminium tube. Thermal conductivity between both was improved with conventional thermal semiconductor paste. The back side of the exchanger was covered with sealing foam for insulation. All the materials were standard in order to obtain an idea about the real possibilities of lowcost adaptation of existing modules. A schematic diagram of the set can be seen in figure 2 .

The exchanger was made with four $40 \times 20 \mathrm{~mm}$ section, 1.3 $\mathrm{mm}$ thick tubes. The two central ones were shorter due to the
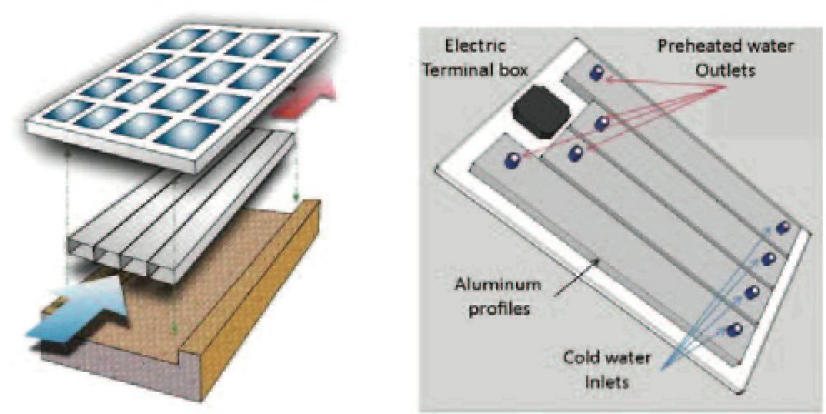

Figure 2. Final prototype: schematic representation of the heat exchanger.

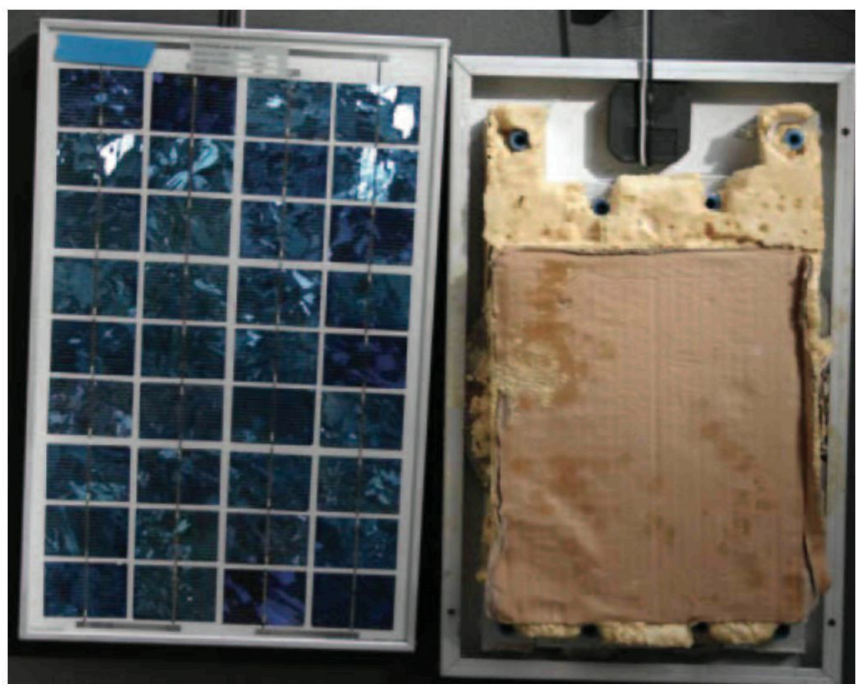

Figure 3. Final prototype. Sun side (left) and back side (right) showing the heat exchanger and insulation.

housing of the module's terminal connection box. The other ones were $40 \mathrm{~cm}$ long. The total area facing the module was approximately $0.058 \mathrm{~m}^{2}$, which shall be assumed its area of contact, although the actual contact between the tubes and the module was not uniform. At both ends of each tube there were valves for the inlets and the outlets as can be seen in figure 3 .

The heat transfer coefficient can be worked out from expression (1), resulting $U \approx 163 \mathrm{~W} / \mathrm{m}^{2} \mathrm{~K}$. As expected, it is a low value, although higher than that measured by Zakharchenko [10].

The aluminium tubes improved on the problem of flatness and evenness of the polyethylene approach, for the sides of the tubes are flat themselves and the only irregularities are the ones from the panel. Also, the aluminium tubes provide both mechanical resistance and high thermal conductivity. The main problem is the contact between tubes and panel, which was minimized with the semiconductor paste. However, it could be observed that the semiconductor paste had not covered the surface completely, leaving gaps of air scattered across. This, apart from reducing $U$, should make it decrease rapidly with the panel temperature, which did in fact happen. 


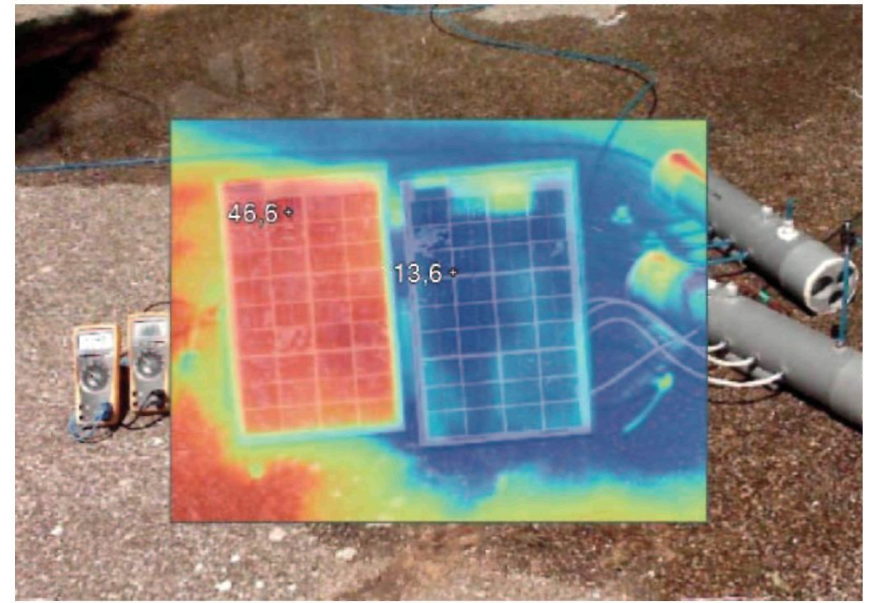

Figure 4. Experimental setup during the preparation of the tests.

\section{EXPERIMENTAL SETUP}

The system was fitted into a small infrastructure formed by inlet and outlet pipes, a stand, a water deposit and a battery of electric resistors. Ambient temperature, $T_{0}$, temperature at the back of the panel, $T_{b}$, inlet, $T_{i}$, and outlet, $T_{o}$, temperatures and volume flow, $i$, were registered for the thermal part; voltage $V$, current $I$ and solar radiation $G$ for the photovoltaic one. A dry (non-cooled) PV identical module was operated beside the previous, so that both received the same radiation. The variables of operation of the dry module were also monitored for comparing both as seen in figure 4 . It can also be observed that the cells nearer to the junction box are have higher temperature than the rest, because they are not in contact with the heat exchanger (see figure 3).

The procedure for each session was as follows:

1) Register ambient temperature and radiation

2) Set a mass flow for the cooled module

3) Start both modules and wait until they are thermally stable

4) Register all variables across the entire range of voltagecurrent pairs $(\mathrm{V}, \mathrm{I})$ starting at $\mathrm{I}=0$ and finishing at $\mathrm{V}=0$

5) Stop. Set a new mass flow and repeat the process while conditions at step 1 hold

A total of 25 sessions were held obtaining measurements for a range of $G$ between 800 and $1096 \mathrm{~W} / \mathrm{m}^{2}$. This text only considers $G \approx 1000 \mathrm{~W} / \mathrm{m}^{2}$.

\section{ENERGETIC PERFORMANCE}

The difference in electrical performance of the dry and two cooled panels can be observed in figure 5. The electrical efficiency of the panel adjusts reasonably well to the correlation shown in equation (3) [1].

$$
\eta_{e}=\eta_{e, T_{r e f}}\left[1-\beta_{T_{r e f}}\left(T_{b}-T_{r e f}\right)\right]
$$

Where $\eta_{e}$ is the electrical efficiency of the panel operating at temperature $T_{b}, \eta_{e, T_{\text {ref }}}$ is its efficiency operating at a reference

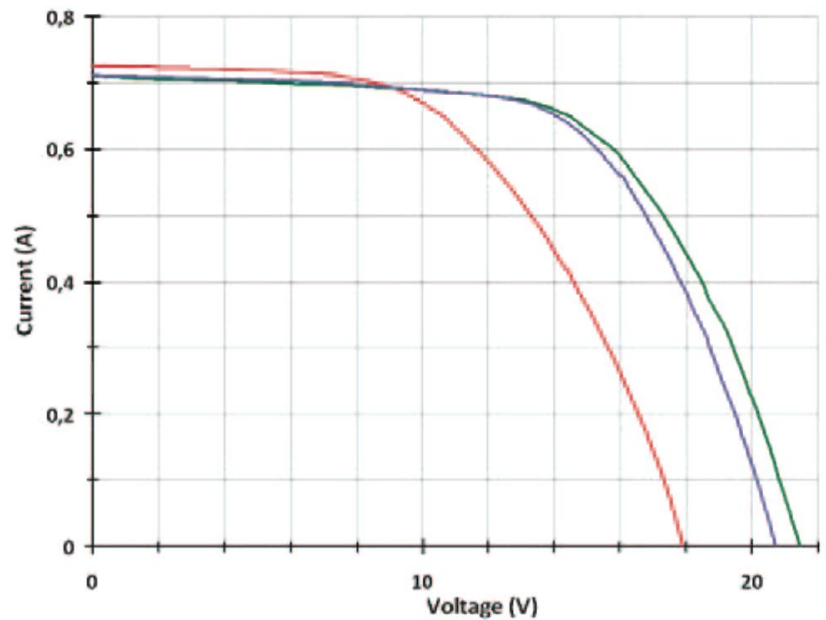

Figure 5. Comparison of the performance of the dry and cooled PV modules for $G \approx 1000 \mathrm{~W} / \mathrm{m}^{2}$.

Table I

EXPERIMENTAL RESULTS FOR POINTS OF MAXIMUM ELECTRICAL POWER AT POINTS OF $T_{b} 22,30,35$ AND $70^{\circ} \mathrm{C}$.

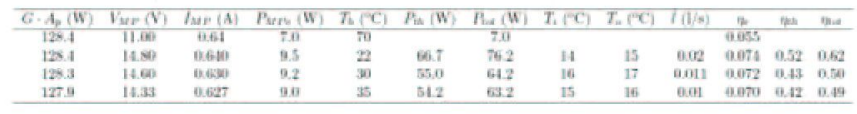

temperature $T_{r e f}$ and $\beta_{\text {ref }}=1 /\left(T_{\text {null }}-T_{\text {ref }}\right)$ is a parameter in which $T_{\text {null }}$ is the temperature at which the efficiency of the panel drops to zero, which is $270^{\circ} \mathrm{C}$ for this kind of panel. In our case $T_{r e f}=22^{\circ} \mathrm{C}, \beta_{r e f}=0.004^{\circ} \mathrm{C}^{-1}$ and $\eta_{e, T_{r e f}}=$ 0.074 . The predicted efficiency of the panel for temperatures $T_{b}$ within 22 and $35^{\circ} \mathrm{C}$ is exact and for $T_{b}=70^{\circ} \mathrm{C}$ it results in a predicted $\eta=0.0597$ against a real $\eta=0.0548$, an overestimation of less that $9 \%$.

Table I shows the readings for the points of maximum electrical power at different temperatures at the back of the panel. The data for three temperatures are shown graphically in figure 6. A clear decreasing trend can be observed in all energetic performances.

Instantaneous thermal efficiency can be expressed in terms of the water inlet reduced temperature [9]: $\Delta T_{R} /\left(G \cdot A_{p}\right)$, being $A_{p}$ the area of panel, where $\Delta T_{R}=T_{i}-T_{0}$. and $G$. $A_{p} \approx 128 \mathrm{~W}$ is the radiation being received by the panel:

$$
\eta_{t h}=0.4107-0.8898 \frac{\Delta T_{R}}{G \cdot A_{p}}
$$

The first term of this expression represents the thermal efficiency when $T_{i}=T_{0}$, and it can be roughly compared to [9] for an order of magnitude, who also built a boxchannel type PVT/w system, resulting differences within $4 \%$. As expectable, the dual, water-air PVT systems described in [14] present higher values in all cases (their lowest is 0.475 for mode $\mathrm{A}$, the simplest configuration). 


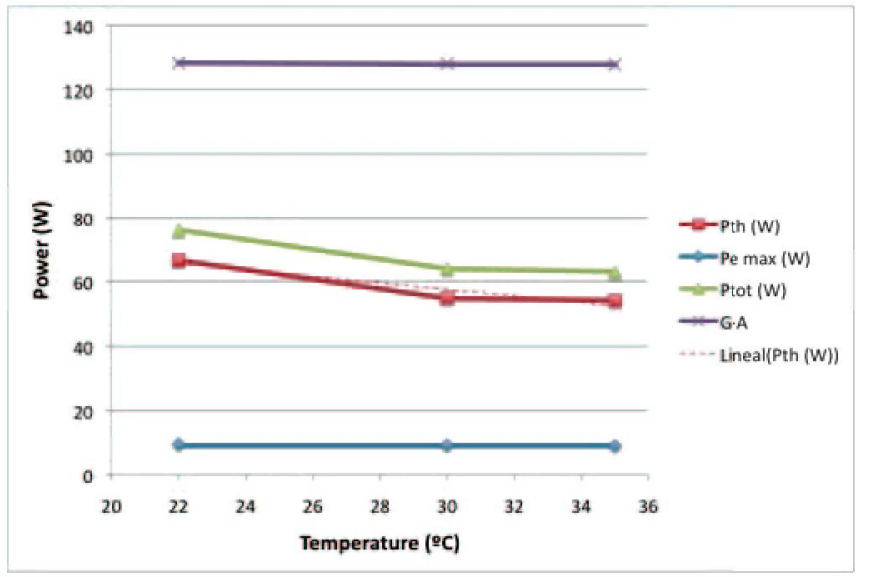

Figure 6. Evolution of total thermal and electrical power with $T_{b}$.

Table II

SCENARIO VARIABLES FOR THE ECONOMIC STUDY.

\begin{tabular}{ll}
\hline Working hours per year & $\mathbf{1 8 2 5}=\mathbf{3 6 5}$ days $\times 5$ hours $/$ day \\
Price of electricity & 0.13 Euro $/ \mathrm{kWh}$ \\
Price of gas & 0.056 Euro $/ \mathrm{kWh}$ \\
\hline
\end{tabular}

\section{CosT}

The purpose of this paper is to obtain a gross estimation of the viability of adapting existing PV panels for PVTw. A basic economic study is necessary to complement the previous technical sections. The focus shall concentrate on its strategical significance more than on the accuracy. The following cases are assessed for achieving a broader perspective:

1) The device analyzed here has been normalized to a $1 \mathrm{~m}^{2}$ panel

2) A cost-optimized version of this extrapolated panel

3) A potential adaptation using commercial Mitsubishi PVTD190MF5

There are two reasons for normalizing to a $1 \mathrm{~m}^{2}$ panel instead of considering the prototype analyzed previously. On one side, this size falls in the trend of commercial panels. On the other, it would allow a quick approximation to estimate the potential of converting an existing field of PV panels by knowing its total area.

The simple payback period, SPP (see equation 5), of the adaptation of the panels shall be calculated as in [8], which is sufficiently representative for the strategic purposes of this text. First estimated the cost of adapting each of the considered PV panels is estimated. Then the increment in electrical and thermal power production that this would yield in the scenario described in table II, which could be representative for Mediterranean countries. The SPP is obtained from here. The results are shown in table III.

$$
\mathrm{SPP}=\frac{\text { Cost of the adaptation }(€)}{\text { extra yearly electric }+ \text { thermal production }(€)}
$$

Table III

COMPARATIVE RESULTS FOR THE SPP FOR EACH OPTION.

\begin{tabular}{cccc}
\hline Panel & $1 \mathrm{~m}^{2}$ & $1 \mathrm{~m}^{2}$ optim. & $\begin{array}{c}\text { Mitsubishi } \\
\text { PV-TD190MF5 }\end{array}$ \\
\hline Adaptation (€) & 345 & 82 & 54 \\
$\Delta P_{e}(€)$ & 5.6 & 5.6 & 9 \\
$\Delta P_{t h}(€)$ & 41 & 41 & 32 \\
SPP (years) & 7.4 & 1.8 & 1.4 \\
\hline
\end{tabular}

In order to interpret the results, it is necessary to explain the considerations made for each of the cases:

- The $1 \mathrm{~m}^{2}$ panel inherits the electrical and thermal efficiencies of the prototype described in the previous sections. Materials and labour have been taken into account in order to estimate the cost of adapting it. It has been assumed that the materials are standard and are paid at the price to the normal consumer. This is representative of manufacturing a very short number of panels in a small workshop.

- The cost-optimized $1 \mathrm{~m}^{2}$ panel assumed the same design and specifications of the previous, but the manufacturing process has been optimized in two ways. First, it is assumed that the materials are not completely standard, but provided by the supplier already cut to the necessary size and applying bulk prices for large series. Second, it is assumed that the technicians are specifically trained and have special tools. This is representative of manufacturing medium-series, which is the natural case of this paper (large series would require a certain extent of automation).

- The commercial Mitsubishi PV-TD190MF5 panel case assumes the same optimized manufacturing process as in the previous case, but design and materials are adapted to its geometry (which implies different arrangement, quantities and cost).

The original panel has $\eta_{e}=0.137$ [15].We have assumed that the refrigerated panel would improve by $20 \%$, reaching $\eta_{e}=0.165$. We have assumed that the thermal efficiency would reach $\eta_{t h}=0.25$. It can be observed that they are significantly more conservative than the ones of our prototype (see table I).

\section{CONCLUSIONS}

This study is a first approach to assess the feasibility of adding water cooling to standard, off-the-shelf PV modules for boosting electrical efficiency and preheating water. Adapting these modules, which have not been specifically designed for PVTw, entails constructive issues which need to be assessed, although energetically they show remarkable costperformance ratio.

The system which was studied here was a prototype based on a small PV module with an ad-hoc elementary aluminium tube heat exchanger added at its back. The system achieved an increase in electrical energy efficiency of $35 \%$ over the nominal, reaching $7.4 \%$ with a $66.7 \mathrm{~W}\left(\approx 520 \mathrm{~W} / \mathrm{m}^{2}\right)$ 
production of thermal energy at $T_{b}=22^{\circ} \mathrm{C}$.

We have verified that thermal contact is the critical factor for performance; both the total transferred heat and the thermal efficiency decrease at higher temperatures of the panel as shown in figure 6, which indicates a poor thermal behaviour.

An interesting aspect to be considered is that a poor exchanger such as the one tested here allows improving the electrical behaviour significantly provided there is abundant water, which might result interesting for some applications. For combined electric-thermal operation, the current exchanger would force operating the device at a low $T_{b}$ to ensure a low $\Delta T_{L M T D}$; a minimal deviation could lead to significant losses in efficiency. A a commercial device should allow a broader range of operation.

In any case it must be remarked that the power and dimensions of the panel tested here do not allow a direct extrapolation of the results to commercial panels for a number of reasons.

The PV module chosen for this experiment had an especially poor performance $\left(\eta_{e} \approx 5.4 \%\right)$; the increase in electrical efficiency in a standard one could be smaller than the one here.

The heat exchangers for full size panels, in the range of 200 $\mathrm{W}$ of electrical power and over one meter long, will imply a more sophisticated support and a different building technique than those used here. This should increase the unit cost, although production in series should lower it. The energetic performance should follow a similar trend to the one shown here, although these values should only be taken as indicative.

A final conclusion from these considerations is that lowcost PVTw systems should be considered as a complementary technology for existing PV plants, water infrastructures and for low power-high autonomy installations.

The reason for this is that they may offer lower total efficiency than specifically designed systems, so that their opportunity comes from operating always at a low $T_{b}$, which equals a small $\Delta T$ in equation (2).

This has two major implications. In terms of thermal energy, they can provide only pre-heating. In terms of electrical energy, operating at a low $T_{b}$ results advantageous for efficiency, so they may improve the electrical performance of existing facilities shortening their payback period at a low cost.

\section{REFERENCES}

[1] E. Skoplaki and J.A. Palyvos. Operating temperature of photovoltaic modules: A survey of pertinent correlations. Renewable Energy, 34(1):23 - 29, 2009.

[2] E. Skoplaki and J.A. Palyvos. On the temperature dependence of photovoltaic module electrical performance: A review of efficiency/power correlations. Solar Energy, 83(5):614 - 624, 2009.

[3] Anja Royne, Christopher J. Dey, and David R. Mills. Cooling of photovoltaic cells under concentrated illumination: a critical review. Solar Energy Materials and Solar Cells, 86(4):451 - 483, 2005.
[4] Anand S. Joshi, Ibrahim Dincer, and Bale V. Reddy. Performance analysis of photovoltaic systems: A review. Renewable and Sustainable Energy Reviews, 13(8):1884 - 1897, 2009.

[5] T. T. Chow. Performance analysis of photovoltaicthermal collector by explicit dynamic model. Solar Energy, 75(2): 143 - 152, 2003.

[6] M. Arif Hasan and K. Sumathy. Photovoltaic thermal module concepts and their performance analysis: A review. Renewable and Sustainable Energy Reviews, 14(7): 1845 - 1859, 2010.

[7] T.T. Chow. A review on photovoltaic/thermal hybrid solar technology. Applied Energy, 87(2):365 - 379, 2010.

[8] Erzat Erdil, Mustafa Ilkan, and Fuat Egelioglu. An experimental study on energy generation with a photovoltaic (pv)-solar thermal hybrid system. Energy, 33(8):1241 $1245,2008$.

[9] Wei He, Tin-Tai Chow, Jie Ji, Jianping Lu, Gang Pei, and Lok shun Chan. Hybrid photovoltaic and thermal solar-collector designed for natural circulation of water. Applied Energy, 83(3):199 - 210, 2006.

[10] R. Zakharchenko, L. Licea-Jiménez, S. A. Pérez-García, P. Vorobiev, U. Dehesa-Carrasco, J. F. Pérez-Robles, J. González-Hernández, and Yu. Vorobiev. Photovoltaic solar panel for a hybrid pv/thermal system. Solar Energy Materials and Solar Cells, 82(1-2):253 - 261, 2004. CANCUN 2003.

[11] PVT ROADMAP A European guide for the development and market introduction of PV-Thermal technology. Technical report, EU PVT Forum, 2006.

[12] Frank P. Incropera, David P. DeWitt, Theodore L. Bergman, and Adrienne S. Lavine. Fundamentals of Heat and Mass Transfer. ISBN: 0471457280. John Wiley \& Sons, 6th edition, September 2006.

[13] T.T. Chow, G. Pei, K.F. Fong, Z. Lin, A.L.S. Chan, and J. Ji. Energy and exergy analysis of photovoltaic-thermal collector with and without glass cover. Applied Energy, 86(3):310 - 316, 2009.

[14] Y. Tripanagnostopoulos. Aspects and improvements of hybrid photovoltaic/thermal solar energy systems. Solar Energy, 81(9): 1117 - 1131, 2007. CISBAT 2005.

[15] http://www.mitsubishielectric.es/archivos/catalogos/ datasheet_pv_module.pdf. 